\title{
Digital control systems for power supplies of space vehicles
}

\author{
Andrey Soldatov *, Juriy Shinyakov, and Maxim Sukhorukov \\ TUSUR, Space Technology Institute, 634050 Tomsk, Russia
}

\begin{abstract}
The principle of building fault-tolerant digital control systems for power supplies of space vehicles is considered in the article. The analysis of the circuits by sampling the controlled component of the output voltage at discrete instants of time is carried out. The possibility of using this approach in multi-channel digital control systems is shown. The results of theoretical studies are confirmed by the example of a four-channel control unit.
\end{abstract}

\section{Introduction}

Widely used analog control systems for switching voltage regulators (SVR) have a number of significant disadvantages: insufficient flexibility in control, impossibility of implementing complex control algorithms, low noise immunity and limited accuracy due to leakage currents, zero-point voltages, temperature of parameter instability, etc.

The use of digital control systems (DCS) improves the parameters of the SVRs, which will provide the following opportunities:

1) selection of the optimal operating modes of the power elements (switching frequency of power switches, change of induction in magnetic elements, current ripple in inductive elements and voltage across capacitors, etc.) [1];

2) the introduction of mathematically complex transfer functions (control laws), their modification without redesigning the equipment;

3) maintenance and issuance of signal information on the load, primary power supply, dangerous operating modes, etc.;

4) realization of intellectual functions of adaptation, self-diagnostics and self-adjustment;

5) forecasting catastrophic regimes;

6) the organization of any interfaces for modern consumers and many other functions [2-4].

Digitization of control algorithm makes it possible to implement the most promising predictive algorithms (predictive control) of frequency control by the pulse width modulation (PWM), which allows reducing the delays in the control loop and the delays of information transfer.

\section{Problem statement}

\footnotetext{
* Corresponding author : soldatov.88@bk.ru
} 
When developing PWM switching voltage regulator in power supply systems (PSS) of space vehicles (spacecraft), the task of researching dynamic characteristics and forming optimal control laws in the "small" signal mode is put on the foreground. Using the approach proposed in [5], it is possible to study dynamics from continuous models, and quantization errors can be estimated from the stationary periodic regime. However, at the synthesis of systems using such continuous models the possibilities of pulse regulation are not used. Other approaches involve taking into account the discrete nature of processes in PWM systems by means of data reduction to the models with pulse-amplitude modulation (PAM) [6], or to the models with a variable structure in the state space [7].

\section{Problem-solving methods}

Of all the known approaches to the synthesis of control laws that ensure high speed with stabilization of the output voltage, a special method is of interest:

- representation of the power circuits of the switching voltage regulators with PWM in the mode of small deviations as an adequate amplitude-impulse model with the controlled components of the processes;

- synthesis of the control law using polynomial equations for the systems with PAM;

- implementation of the synthesized control law in switching voltage regulator taking into account specifics of PWM.

This method allowed its authors to synthesize a control law that provides the minimum finite duration of transient processes in the switching voltage regulator based on DC-DC buck converter. Such a law is called optimal by the authors for speed, and a voltage regulator with such a control law is a high-speed voltage regulator. In the proposed approach to the synthesis of the control law was further developed for both boost and buckboost DC-DC converters.

In accordance with the discrete transfer function of the synthesized correction device and taking into account the specifics of PWM, two ways to implement the PWM control device are proposed:

- on instantaneous values of state variables;

- on the values of state variables, selected at discrete instants of time.

$$
W_{\mathrm{K}}(p)=d+d\left(1-e^{-p T}\right)
$$

where $d=L C / T, L$ and $C$ are the inductance and capacitance of the output filter of switching voltage regulator.

The implementation of the $P W M$ control law, in which only discrete values of the regulated component of the voltage across the capacitor of the output filter are used, is shown in Fig. 1.

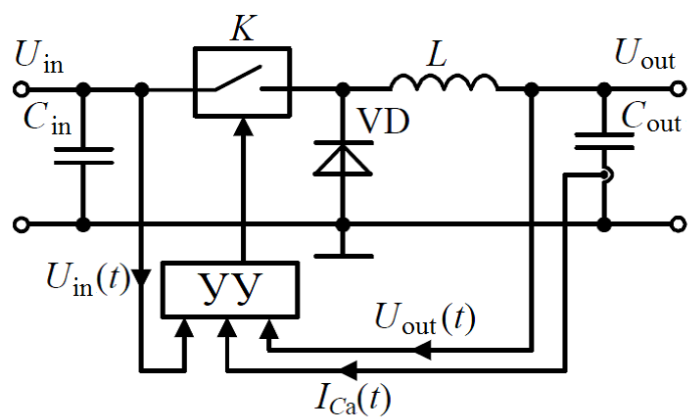

Fig 1. Switching voltage regulator with discrete control 
In this scheme, the increment of the input signal of the pulse-width modulator is determined from the expression:

$$
\Delta U_{y}(m T)=-\frac{d_{0}}{U_{i n} K_{m}}\left[2 \Delta U_{C p}(m T)-\Delta U_{C p}(m-1) T\right],
$$

where $U_{i n}$ is the voltage at the input of the scheme; $K_{m}=\Delta t_{c . p} / \Delta U_{y}(m T), \Delta t_{c . p}$ increment of the pulse duration of the control pulse of the power switch of the regulator with respect to the time $m T$ of the controlled switching; $\Delta U_{y}(m T)$ is the increment of the $P W M$ input signal; Um is the amplitude of the sawtooth PWM voltage, $T$ is the conversion period.

Given the condition $\Delta t_{c . p}<T$, it can be assumed that the controlled switching of the power switch of the voltage regulator occurs with a period of $T$.

The current values of the dynamic component of the input signal of the pulse-width modulator are given by:

$$
U_{y}(m T)=U_{y}((m-1) T)+\Delta U_{y}(m T)
$$

It is impossible to determine the regulated component of the voltage $U_{C p}$ across the capacitor of the output filter of the regulator by performing computational operations with discrete or continuous values of the output voltage $U_{\text {out }}$ of the scheme. This is explained by the fact that the equivalent circuit for the capacitor of the output filter can be represented as a series connection of capacitance $C_{f}$ and internal resistance $R_{C}$ of a capacitor. Therefore, the output voltage of regulator which is the voltage across the capacitor of the output filter of scheme consists of the voltage across the capacitance $U_{C}$ and the voltage across the internal resistance $U_{R c}$.

Since the internal resistance $R_{C}$ of the capacitor can significantly change under the influence of temperature and time factors, then the voltage $U_{R c}$ will also change, and therefore, to determine the voltage across the capacitance $U_{C}$ for example, by subtracting the undefined voltage $U_{R c}$ from the output voltage $U_{o u t}$ is not possible.

It is possible to calculate the increment of the regulated component of the voltage $\Delta U_{C p}$ across the capacitance over the period $T$ by integrating the increment of the regulated component of the capacitor current $\Delta I_{C p}$ in the time interval equal to the period $T$ :

$$
\Delta U_{C p}(m T)=\frac{1}{C} \int_{(m-1) T}^{m T} \Delta I_{C p}((m-1) T) d t,
$$

Since the increment of the regulated component of the capacitor current in the interval between the adjustable switching times $m T$ of the power switch of the voltage regulator 
remains unchanged (Fig. 1), then to determine it the first difference in the capacitor current should be calculated:

$$
{ }^{\Delta I_{C p}}(m T)=I_{C p}(m T)-I_{C p}((m-1) T)
$$

or

$$
{ }^{\Delta I} C_{C p}(m T)=I_{C p}(m T+\tau)-I_{C p}((m-1) T+\tau),
$$

where $\tau<T$ is, in general case, an arbitrarily chosen fixed time interval.

In order to have the time for carrying out the computational procedures necessary to determine the input signal of the pulse-width modulator at the instant of time $m T$, it is advisable to select $\tau$ such a way that the moments of time $(m-1) T+\tau$ are as far removed from the moments $m T$ of the adjustable switching of the power switch of voltage regulator. For DC-DC buck converter and for modulation of the fall time of the pulse, the time instants $(m-1) T+\tau$ should be selected immediately after the power switch is turned on.

As a rule, the static duty cycle $K_{S t}>0.25$ in the buck DC-DC converters, therefore, the sampling time of the discrete values of the input signals and the computation is still close to a quarter of the conversion period. Using expression (1.5) allows us to define ${ }^{\Delta} I_{C p}(m-1) T+\tau$ at time $(m-1) T+\tau$.

Since the increments of the regulated component of the voltage in the interval between the adjustable switching times of the power switch of the voltage regulator are constant, then (1.4) can be written as:

$$
\Delta U_{C p}(m T)=\frac{1}{C} \Delta I_{C p}((m-1) T),
$$

Thus, replacing the integration procedure in accordance with (1.4) by determining the area of the rectangle according to (1.6) allows us to determine the increment of the regulated component of the voltage across the capacitance $\Delta U_{C p}(m T)$ in the vicinity of the time moment $(m-1) T+\tau$, i.e. before the moment of time $m T$, in the neighborhood of which the adjustable edge of the control pulse of the power switch is formed. Accordingly, the calculation of the dynamic component of the input signal of the pulse-width modulator in accordance with (2) and (3) can also be performed in the vicinity of the time instants $(m-1) T+\tau$.

\section{Practical implementation}

Figure 2 shows the block diagram of the control device which implements the discrete law (1) of the input PWM signal. 


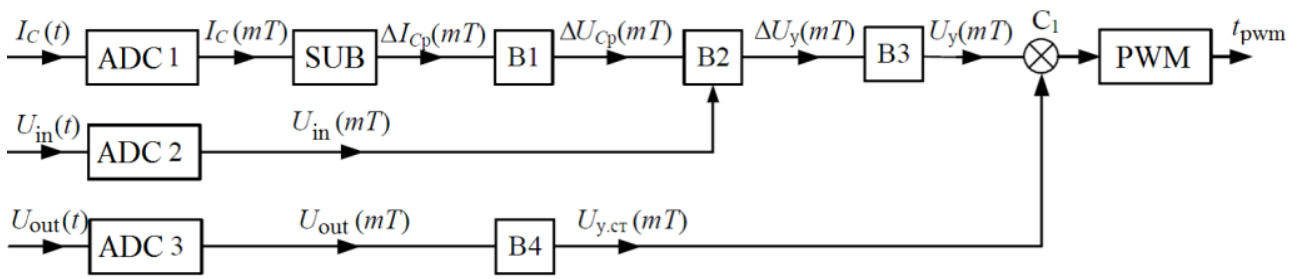

Fig. 2. Block diagram of microprocessor control device

Analog-to-digital converters ADC1 ... ADC3 provide sampling of input signals at time moments $m T+t_{O F F}$, where $t_{O F F}$ is the offset interval and digitization of selected signal values at subsequent time intervals with a duration in the period of sampling $T$. The first substrator provides (5), and calculators $B 1, B 2$ and $B 3$ perform calculations according to (6), (2) and (3).

\section{Conclusion}

The considered variant of realization (1) with application of discrete values of a regulated component of an output voltage can be used to control power modules (PM), which are the part of multimodular switching voltage regulator. The control device for PM, which is a part of a multimodal switching regulator, in addition to the tasks to ensure the required quality of the output voltage in dynamic and static modes of operation, must also solve the problem of distributing the load current between the individual modules included in the regulator. As a rule, the developers seek to ensure balancing distribution of the load current between the PMs, which allows to equalize the heat dissipation in the modules. For an even distribution of the load current between the PMs in the analog realization of the control device, a negative feedback loop (FL) is often used for the inductor current. The application of this circuit forms an oblique external characteristic of the module, which helps to balance the output currents of the PM.

In the course of tests to provide the functions (logic of operation) and the requirements of operability (fault tolerance), it was established that the layout of the digital control module completely confirmed the declared technical characteristics.

The research was made to implement decree of the Government of the Russian Federation of 9 April, 2010 No. 218 and contract between ISS JSC and the Ministry of Education and Science of the Russian Federation of 01 December 2015 No. 02.G25.31.0182.

\section{References}

1. O. A. Kozhemyak, S. K. Zeman, E. V. Yaroslavtsev, EDM, 236 (2004)

2. H. Liu, C. Fu, N. Li, A. Soldatov., W. Han, MATEC Web of Conf., (2017)

3. E. Y. Burkin, O. A. Kozhemyak, Inst. Exp. Tech, 59, 245 (2016)

4. V. D. Yurkevich, SIBIRCON, 124 (2017).

5. H. Bodur, A. F. Bakan, M. Baysal, El. Eng., 85 (1), 45 (2003)

6. M. Borage, S. Tiwari, S. Kotaiah, IEEE T. on I. Electr., 52 (6), 1547 (2005)

7. Y. A. Shinyakov, V. D. Semenov, V. A. Kabirov, D. S. Torgaeva, M. P. Sukhorukov and R. S. Cevastyanov, SIBIRCON, 346 (2017) 